at the posterior wound is a massive proliferative choroiditis observed through a large retinal hole.

We wish to thank Col. J. Biggam, M.C., Commanding (Scottish) General Hospital and Brigadier G. I. Scott, Consultant Ophthalmologist, Middle East Forces, for their kind permission to publish these notes. The Micro-photographs were done in the Hadassah Hospital, Jerusalem. We are grateful to the authorities and to Professor Feigenbaum who made the necessary arrangements. The fundal paintings were done by Dr. J. Kraus.

\title{
SUB-HYALOID HAEMORRHAGE FOLLOWING "T.A.B." INOCULATION
}

BY

\author{
Major J. P. F. LLOYD, R.A.M.C.
}

IT is well known that spontaneous haemorrhages, sometimes severe, may occur in any part of the body following routine immunization with T.A.B. Little is known of the aetiology of this condition; it is suspected that it may be due to a temporary increase in capillary permeability. The following case is reported because it is believed that haemorrhage in the eye has not previously been recorded in this association.

A man aged 36 years, of normal physique and previous good health received his routine injections of T.A.B., the first of 500 millions on February 15 , and the second of 1,000 millions on March 2. Each injection produced a normal reaction. On March 3 he noticed that the vision of the Yeft eye was extremely poor but he did not report sick until some days later.

When examined on March 16, the right eye was normal, having a visual acuity of $6 / 6$. The left eye could only discern hand movements, and an extensive sub-hyaloid haemorrhage was found lying in front of the macular region, to the temporal side. $\mathrm{He}$ was put completely at rest and atropine drops were instilled in the affected eye.. The haemorrhage began to clear slowly, retinal vessels on the temporal side being visible five days later through the vitreous haze.

General investigation showed some chronic bronchitis; the blood. picture was normal. When last seen by me on April 2, absorption of the haemorrhage was continuing but vision was still hand movements only. I am indebted to Major E. F. King, R.A.M.C., for a later report from a convalescent hospital on June 3, which states that "there was still considerable unabsorbed haemorrhage in the left vitreous." 